\title{
Reflexión sobre prácticas centradas en la familia desde la perspectiva de estudiantes de fisioterapia, terapia ocupacional y logopedia del sureste de España
}

\author{
Reflection on Family-Centered Practices From the Perspective of Students of Physical \\ Therapy, Occupational Therapy, and Speech Therapy in the Southeast of Spain
}

\section{Reflexão sobre práticas centradas na família a partir da perspectiva de estudantes de fisioterapia, terapia ocupacional e fonoaudiologia do Sudeste de Espanha}

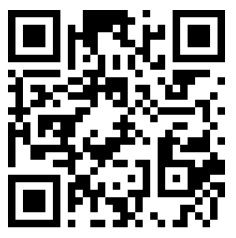

Noelia Orcajada-Sánchez

Universidad de Murcia

Murcia, España

noelia.orcajada@um.es

(iD) https://orcid.org/0000-0003-3476-9291

Elisabet Ortuño-Cano

Equipo InSitu

Alicante, España

elifisiopeques@gmail.com

(iD) https://orcid.org/0000-0002-9806-7590

Conchi Martínez-Pérez

Equipo InSitu

Alicante, España

conchi8_0212@hotmail.com

iD https://orcid.org/0000-0002-6575-8512

Francisco Alberto García-Sánchez

Universidad de Murcia

Murcia, España

fags@um.es

iD https://orcid.org/0000-0003-3788-6354

Recibido • Received • Recebido: 04 / 02 / 2019

Corregido • Revised • Revisado: 30 / 03 / 2020

Aceptado • Accepted • Aprovado: 07 / 07/ 2020 
http://doi.org/10.15359/ree.24-3.6

http://www.una.ac.cr/educare

educare@una.cr

Resumen: Cada vez son más las personas profesionales de atención temprana que basan su labor en unas prácticas centradas en la familia. El cambio en esta práctica profesional hace que cuando en la docencia universitaria se empieza a abordar esta temática, aunque solo sea de forma puntual, se generen importantes dudas en el alumnado, más habituado a prácticas ambulatorias. En este artículo, analizamos las dudas planteadas por estudiantes de los casos de los grados de Fisioterapia, Terapia Ocupacional y Logopedia de dos universidades diferentes del sureste de España, cuando un equipo profesional de atención temprana les habla de estas prácticas centradas en la familia. El profesorado de algunas asignaturas impartidas en estos grados organizó unas acciones formativas sobre las prácticas centradas en la familia dentro de sus asignaturas. A través de un diseño no experimental, cualitativo, con metodología observacional, describimos las dudas planteadas por 196 estudiantes asistentes a dichas acciones formativas. Recogemos, también, las respuestas a cada una de esas dudas, consensuadas por un grupo de profesionales de estas prácticas. Entendemos que son preguntas que pueden surgir a profesionales o estudiantes, dado que el proceso por el que atraviesan para entender y adoptar las prácticas centradas en la familia suele ser siempre similar, por lo que resulta útil su conocimiento y divulgación. Nuestros resultados señalan diferencias en las dudas planteadas por el alumnado de los diferentes grados, las cuales pueden reflejar tanto diferencias en su formación clínica como en la formación previa recibida en atención temprana. Se defiende la importancia de esta formación en futuros estudiantados egresados de titulaciones que les habilitan para ejercer su labor en atención temprana.

Palabras claves: Atención temprana; desarrollo profesional; intervención centrada en la familia; formación.

Abstract: More and more early childhood professionals are basing their work on family-centered practices. The change in this professional practice means that when university teaching begins to address this matter, even if only occasionally, it generates significant doubts in students, who are more accustomed to outpatient practices. In this article, we analyze doubts raised by students when an early childhood professional team talked to them about these family-centered practices. These doubts were about cases studied in Physiotherapy, Occupational Therapy, and Speech Therapy programs from two different universities in southeastern Spain. Some teaching staff members in these degrees organized training actions on family-centered practices in their courses. Through a non-experimental, qualitative design with an observational methodology, we described the doubts raised by 196 students attending these training actions. We also collected the answers, agreed upon by a group of professionals of these practices, to each of these doubts. We understand that these are questions that may arise to professionals or students, given that the process they go through to understand and adopt family-centered practices is usually similar, so it is useful to know and disseminate them. Our results point to differences in the questions raised by students of the different programs; they may reflect differences in their clinical training and in the previous training received in early childhood programs. We defend the importance of this training in future graduates from these degrees that qualify them to work as early childhood professionals.

Keywords: Early care; family-centered practices; professional development; training. 


\begin{abstract}
Resumo: Cada vez mais profissionais de cuidados precoces estão a basear o seu trabalho em práticas centradas na família. A mudança nesta prática profissional significa que quando o ensino universitário começa a abordar esta questão, mesmo que apenas ocasionalmente, gera dúvidas significativas nos estudantes, que estão mais acostumados a práticas ambulatoriais. Neste artigo, analisamos as dúvidas levantadas por estudantes dos casos de Fisioterapia, Terapia Ocupacional e Fonoaudiologia de duas universidades diferentes do sudeste da Espanha, quando uma equipe profissional de atendimento precoce fala com eles sobre estas práticas centradas na família. O corpo docente de algumas disciplinas leccionadas nestas licenciaturas organizou algumas acções de formação sobre práticas centradas na família dentro das suas disciplinas. Através de um desenho não experimental e qualitativo com metodologia observacional, descrevemos as dúvidas levantadas por 196 alunos que participaram destas acções de formação. Também recolhemos as respostas a cada uma destas dúvidas, acordadas por um grupo de profissionais destas práticas. Entendemos que estas são questões que podem surgir para profissionais ou estudantes, dado que o processo pelo qual passam para compreender e adotar práticas centradas na família é geralmente sempre semelhante, por isso é útil conhecê-las e divulgá-las. Os nossos resultados apontam para diferenças nas questões levantadas pelos alunos nas diferentes séries, que podem reflectir tanto diferenças no seu treino clínico como no treino anterior recebido nos cuidados iniciais. Defendemos a importância desta formação em futuros licenciados que os qualifica para trabalharem em cuidados precoces.
\end{abstract}

Palavras-chave: Cuidados precoces; desenvolvimento profissional; intervenção centrada na família; formação.

\title{
Introducción
}

Durante las últimas décadas, tanto a nivel nacional como internacional, muchas personas profesionales de atención temprana han pasado a desarrollar su trabajo desde la perspectiva de unas prácticas de intervención centradas en la familia (PCF). Estas prácticas suponen una nueva filosofía de base para profesionales que desempeñan su labor en este campo, quienes necesitarán una formación específica, tanto a nivel técnico como socioemocional (García-Sánchez et al., 2018). No sólo deberá dominar la evolutiva de la niñez y las características de las distintas discapacidades y alteraciones del desarrollo, importante para conocer su evolución; y conocer los objetivos y estrategias de intervención de las diferentes disciplinas, para facilitar el trabajo en equipo (Federación Estatal de Asociaciones de Profesionales de Atención Temprana [GAT], 2000; Ruiz-Veerman et al., 2005). También deberá dominar una serie de prácticas relacionales, que le permitan una interacción adecuada con la familia; y unas prácticas participativas, para favorecer en la familia una implicación activa en la intervención de su hijo o hija (Dunst, 2000; 2002).

Es evidente que profesionales que trabajan en atención temprana provienen de distintos ámbitos (psicología, educación, fisioterapia, logopedia, etc.). Por ello, se hace necesaria una formación en materia de atención temprana que sea común para quienes, aun teniendo una titulación de base proveniente de diferentes disciplinas, quieran especializarse en este campo (García-Sánchez et al., 2018; GAT, 2000; Orcajada Sánchez, 2017; Ruiz-Veerman et al., 2005). 
http://doi.org/10.15359/ree.24-3.6

http://www.una.ac.cr/educare

educare@una.cr

Ahora bien, en nuestro país, todavía hay una tradición, muyarraigada, quelleva a desempeñar una intervención clínica y ambulatoria dentro de la disciplina de la atención temprana. Esto deriva en que, todavía, mucha de la docencia impartida siga formando en este enfoque tradicional de la disciplina y ofrezca, al estudiantado cercano a egresar, una visión profunda de ese enfoque clínico, muchas veces olvidando y obviando la amplia evidencia científica de la que gozan las PCF. Actualmente, existen numerosos documentos y estudios que avalan el importante quehacer de profesionales de atención temprana que desempeñan su labor basándose en unas PCF. Estos documentos dejan constancia de la evidencia científica que poseen estas prácticas (Dunst, 2017; Forry et al., 2011; García-Sánchez et al., 2014; Dunst et al., 2007).

Sin embargo, a pesar de esa abultada evidencia científica de la que hablamos, son fuertes las raíces de la perspectiva ambulatoria clínico/rehabilitadora en atención temprana desde sus inicios. Ello hace que los nuevos egresados de la universidad con titulaciones que les habilitan para ejercer su labor en atención temprana se cuestionen ciertas ideas y duden de algunos postulados que se les plantean cuando se enfrentan a la filosofía subyacente a las PCF, los beneficios que estas tienen para el niño, la niña y su familia y, sobre todo, la forma de poner en marcha estas prácticas.

En este trabajo pretendemos reflejar, mediante un análisis descriptivo y cualitativo, algunas de las dudas planteadas por estudiantes de los grados de Fisioterapia, Terapia Ocupacional y Logopedia de dos universidades diferentes, tras recibir la misma acción formativa sobre las PCF. Además de ello, puesto que el fin último de estas charlas era contribuir a la formación de los futuros sujetos profesionales en materia de atención temprana, y concretamente, de las PCF, recogemos también, en este trabajo, las respuestas dadas a cada una de las preguntas más habituales que planteó el alumnado al finalizar la charla. De esa manera, esperamos contribuir a que estén disponibles para cada estudiante y profesional que necesite una respuesta al respecto, así como para los propios grupos formadores de profesionales de esta área.

\section{Método}

\section{Diseño de investigación}

Diseño no experimental, con enfoque puramente cualitativo, llevado a cabo como un estudio descriptivo con una metodología observacional. A través de esta metodología se describen las dudas, sobre la intervención centrada en la familia en cuanto a la atención temprana, planteadas por una muestra no probabilística, seleccionada por conveniencia, constituida por alumnado voluntario de los grados de Fisioterapia, Terapia Ocupacional y Logopedia de dos universidades del sureste de España. También siguiendo un enfoque cualitativo, se describen las respuestas a esas dudas, previamente trianguladas por un equipo de profesionales de la disciplina con experiencia en estas practicas de intervención. 
http://doi.org/10.15359/ree.24-3.6

\section{Participantes}

Participaron en este estudio un total de 196 estudiantes de los grados en Fisioterapia, Terapia Ocupacional y Logopedia de dos universidades del sureste de España, quienes se convocaron por su profesorado, a una acción formativa consistente en una charla impartida por profesionales de atención temprana en ejercicio que implementan PCF. En concreto:

- 90 estudiantes de Fisioterapia en Especialidades Clínicas VI (Fisioterapia en Pediatría) del segundo semestre de $3^{\circ}$ curso del Grado en Fisioterapia de la Universidad Miguel Hernández.

- 40 estudiantes de la asignatura Terapia Ocupacional en la Infancia de $2^{\circ}$ curso del Grado en Terapia Ocupacional de la Universidad Miguel Hernández.

- 66 estudiantes de la asignatura Desarrollo Profesional de $4^{\circ}$ curso del Grado en Logopedia de la Universidad de Murcia.

En ninguno de estos grados existía una asignatura específica e íntegra sobre PCF en atención temprana, debido a lo cual una parte del profesorado propone introducir algún tema sobre estas prácticas dentro de sus asignaturas.

Es importante señalar que el alumnado, tanto del Grado en Fisioterapia como del Grado en Terapia Ocupacional, tuvo un acercamiento a las PCF dentro de las asignaturas en las que se impartieron las charlas y previo a ellas, pero a nivel teórico y de manera superficial. Ninguno de estos grados cuenta con una asignatura específica de atención temprana en su plan de estudios. En cuanto al Grado en Logopedia, este estudiantado tuvo un acercamiento más profundo a las PCF dentro de una asignatura específica de Atención Temprana que cursaron como obligatoria en el curso anterior ( $3^{\circ}$ curso).

\section{Procedimiento}

En las distintas titulaciones en las que se ha llevado a cabo la recogida de información se desarrolló la misma acción formativa de, aproximadamente, dos horas de duración, consistente en una charla interactiva sobre Prácticas de atención temprana centradas en la familia. La acción formativa corrió a cargo de tres profesionales del Equipo InSitu (de Crevillente, Alicante): una fisioterapeuta, una psicóloga y una profesional con el doble perfil de pedagoga y logopeda; todas ellas formadas en prácticas de intervención centradas en la familia, las cuales implementan en su desempeño laboral. Durante la acción formativa, el estudiantado ya podía formular sus dudas o comentarios, aunque la mayoría esperó al final para hacerlo, se dedicaron unos treinta minutos para tal fin. 
http://doi.org/10.15359/ree.24-3.6

http://www.una.ac.cr/educare

educare@una.cr

En cuanto a los contenidos que se plantearon en esta acción formativa, estos fueron los siguientes:

- Nuestros inicios como equipo

- Modelo de intervención centrado en la familia vs PCF

- El equipo transdisciplinar

- El rol de cada profesional en las PCF

- El papel de las familias en estas prácticas

- Herramientas de valoración e intervención para unas PCF

Estos contenidos fueron acompañados del visionado de algún vídeo en los Grados de Terapia Ocupacional y Logopedia, estos vídeos se utilizaron concretamente de la siguiente manera:

- Grado de Fisioterapia, no hubo posibilidad de proceder al visionado de vídeos por motivos técnicos.

- Grado de Terapia Ocupacional, se visionó un vídeo al finalizar los contenidos de la acción formativa y previo al turno de preguntas. En este vídeo aparecía una familia contando lo que había supuesto para ella el trabajo en estrecha colaboración de igual a igual con el profesional, desde unas PCF, y la evolución que apreciaban, en su hijo y en la familia en general, desde los inicios de la intervención.

- Grado en Logopedia, se hizo uso del vídeo anterior más otro vídeo en el que aparecía una profesional haciendo un entrevista basada en rutinas a una familia.

En cada una de las titulaciones se recogieron todas las preguntas o comentarios que surgieron entre estudiantes que participaron en cada una de ellas. Para esa recogida de información se estableció un procedimiento en dos fases. En primer lugar, en todas las charlas realizadas, una de las profesionales que las impartieron asumía la responsabilidad de registrar literalmente las preguntas o comentarios del alumnado. En segundo lugar, finalizada la charla, las tres profesionales implicadas repasaban el listado de preguntas registradas para corroborar su exactitud.

Se consideraron para este estudio todas las preguntas que se realizaron, si bien se aglutinaron en una sola aquellas que, formuladas por el mismo grupo de estudiantes, coincidían en contenido, aunque se hubieran realizado de manera algo diferente. Solo se excluyeron aquellas preguntas que no hacían referencia a las prácticas de intervención centradas en la familia, como fue el caso de preguntas referidas a características de la población a atender, a la formación necesaria para trabajar en la disciplina o a la forma de acceso a este campo laboral. 
http://doi.org/10.15359/ree.24-3.6

Las respuestas que se presentan en los resultados del presente estudio resumen la argumentación dada en el momento de la realización de la pregunta, así como las consideraciones posteriormente planteadas en la reflexión conjunta y puesta en común entre el equipo autor de este estudio. De esta forma, las respuestas presentadas han sido consensuadas por cuatro profesionales, con perfiles disciplinares diferentes (fisioterapia, logopedia, psicología y pedagogía), la totalidad con formación y experiencia profesional en prácticas de intervención centradas en la familia en atención temprana.

\section{Resultados}

Las preguntas realizadas por cada uno de los grupos de estudiantes presentaban diferencias en cuanto a sus centros de interés. A continuación, recogemos las preguntas registradas, una vez eliminadas aquellas que no hacían referencia a las prácticas de intervención centradas en la familia, y señalamos la respuesta aportada a cada una de las dudas y cuestiones planteadas. De esta manera, esperamos contribuir a la formación de profesionales de atención temprana, tanto de quienes que ya se encuentran en activo, como aquellos otros grupos que todavía están formándose y quieran adoptar en su quehacer laboral las PCF. Igualmente, estas respuestas pueden se de utilidad para el personal formador de estos grupos de profesionales.

En el Grado en Fisioterapia, las dudas se centraban en las dificultades que tendrían los padres y madres a la hora de poner en marcha el proceso de intervención con su hijo o hija, puesto que, según el estudiantado, este nunca lo haría como profesional. Concretamente, las preguntas planteadas por este grupo de estudiantes fueron del tipo:

- Si la familia no hace de forma correcta una técnica de intervención, ¿qué hacemos como profesionales? ¿Va a ser efectivo? Un padre o madre no va a reproducir una técnica de fisioterapia como un profesional.

Respuesta: NO se trata de convertir a los padres o madres en terapeutas, ellos son padres y madres, y queremos que sigan siéndolo por encima de todo. Nuestra meta no es que reproduzcan técnicas. Debemos conseguir que identifiquen estrategias que puedan usar en su día a día y que sean las más adecuadas para mejorar la participación y la actividad del niño o la niña, de la manera más funcional posible. Por supuesto, la persona cuidadora principal no lo va a hacer nunca como profesional, pero es que nunca pretendemos que la familia sea terapeuta. No pretendemos que la familia reproduzca la forma de hacer ni que desarrolle una intervención técnica como podría hacer el sujeto profesional. Nuestro objetivo, como profesionales, es que los sujetos cuidadores principales de la niñez, quienes comparten más tiempo con él o ella, sepan identificar sus necesidades y sepan darles respuestas adecuadas en su vida diaria. Buscamos que sepan aprovechar las oportunidades de aprendizaje que les brinda su día a día, en su entorno natural. Para 
ello no es necesario ni aconsejable que reproduzcan técnicas, sino que identifiquen sus propias herramientas y estrategias, y sepan darle uso, que sepan utilizarlas.

- Si la familia no plantea hacer lo mejor para su hijo, o no se implica, ¿qué haces, lo dejas, aunque sepas que no es lo mejor?

Respuesta: Siempre lo ven, nunca nos hemos encontrado una familia que, con ajustes de sus expectativas tras facilitarles la información pertinente, no vea "lo importante" $y$ cómo ayudar a su hijo o hija. Creer que no es así es más un prejuicio profesional que una realidad. Una madre, un padre, cualquier miembro de la familia, siempre quiere lo mejor para ese niño o esa niña. De hecho, si no fuese así, tampoco buscaría ni solicitaría nuestra ayuda. Es cierto que muchas veces la familia no se termina de implicar como esperamos que lo hiciese. En esos casos, habrá que analizar, con la familia, por qué es así. Para ello basta preguntarnos, o mejor, preguntarle a la familia, qué espera de nuestra intervención, cuáles son sus preocupaciones, cuáles son sus necesidades...y, a partir de ahí, ayudarles a marcarse objetivos y a cumplir expectativas, identificando estrategias y cómo ponerlas en marcha. No se trata de imponer a la familia lo que debe hacer, ya que, de hacerlo así, la familia no se implicaría. Nosotras, como profesionales, no estamos viviendo la situación por la que esa familia atraviesa cada día. Se trata de entender su situación, de ponernos en su lugar y apoyarles y ayudarles, analizando el punto en el que se encuentran y hasta dónde quieren llegar.

- Dejas de tratar a la niña o al niño porque trabajas con la familia, ¿entonces no le valoras ni le tocas?

Respuesta: El sujeto profesional sigue siendo fisioterapeuta pediátrico o la titulación que tenga, con su formación en valoración e intervención, la cual debe seguir usando. Pero, a la vez, añades otras herramientas de valoración como las de participación, autonomía, escalas de calidad de vida familiar... Hasta ahora la persona profesional, sea fisioterapeuta, logopeda, psicóloga o el perfil profesional que tenga, ha sido la única experta. Tenía el conocimiento técnico y era la única que sabía cómo hacer. Además de ese conocimiento técnico ha sabido utilizar muy bien las prácticas relacionales para que la familia confiara e, incluso, le proporcionara toda la información necesaria. Pero cuando trabajamos desde unas PCF, también tenemos que hacer uso de unas prácticas participativas (más allá de las relacionales). Esas prácticas participativas son las que van a favorecer la implicación de la familia en el proceso de intervención. Y es en esas prácticas participativas en las que el sujeto profesional ya no ha sido tan hábil, aunque estamos aprendiendo a utilizarlas. Todo esto no significa que el sujeto profesional no necesite ni utilice sus conocimientos técnicos que le proporciona la disciplina en la que se ha formado. Todo se va sumando y va complementando nuestra labor como profesionales de AT. Lógicamente, cuando es necesario valorar y tocar directamente 
http://doi.org/10.15359/ree.24-3.6

al niño o a la niña, lo haremos. No olvidemos que nuestro objetivo de intervención sigue siendo la población infantil, pero ahora lo es a través de la familia. En ese sentido, nunca dejaremos de tratar a la niñez, solo que ahora no lo hacemos directamente, sino a través de nuestra aliada principal, la que le mejor conoce: su familia.

- Si existe un problema estructural, ¿lo dejas de tratar?

Respuesta: No dejas de tratarlo, si es necesario. Pero, al principio pensamos que todo lo debemos tratar y no es así. Si el niño o la niña necesita un control postural la familia lo verá y lo pedirá. Y si no fuera el caso, al principio, nuestra labor será hacerles reflexionar para que lleguen a valorarlo. No tiene ningún sentido tratar un determinado problema, aunque sea estructural, durante una sesión directa con el niño o niña, si el resto del tiempo, a lo largo del día y de la semana, no se va a hacer nada por seguir tratándolo. El sujeto profesional no puede estar corrigiendo la postura, por ejemplo, todas las horas del día en que sea necesario, los 7 días de la semana. Sin embargo, si hacemos competente a la familia en ese control postural, la familia sí tendrá muchísimas más oportunidades para intervenir o corregir esa postura al cabo del día. Le debemos honestidad a nuestra propia persona y a la familia al plantear lo que somos y no somos capaces de hacer.

- Vemos estas prácticas más como un complemento a la intervención clínica que como única opción.

Respuesta: La evidencia nos dice que estas prácticas son más eficaces. Tenemos revisiones sistemáticas y estudios de metaanálisis que avalan la evidencia científica de las PCF, tanto directamente como de forma indirecta, garantizan mayor evidencia a favor de estrategias de intervención en el entorno natural, a través de personas cuidadoras principales y objetivos funcionales. Sin embargo, no existe estudio de ese nivel que avale evidencia científica de aquellas intervenciones propias de un modelo clínico/ ambulatorio. ¿Tiene sentido seguir poniendo en práctica una forma de intervención de la que no tenemos constancia de sus efectos cuando sí existen otras prácticas cuyos beneficios están avalados científicamente?

En el Grado en Terapia Ocupacional las dudas versaron, sobre todo, en torno al equipo transdisciplinar y el planteamiento de los objetivos:

- ¿Dónde quedan las competencias de cada profesional? Cada perfil tiene sus competencias y creemos que otro no puede hacer nuestro trabajo.

Respuesta:Las PCF sebasan en el trabajo en un equipo transdisciplinar. Por ello, en lo relativo o perteneciente a una transferencia de información, conocimientos o técnicas, cada miembro del equipo trata de ampliar la información, así como los conocimientos y competencias de sus pares. Se trata de un aprendizaje en equipo, donde cada profesional trata de planificar y 
apoyar la enseñanza-aprendizaje de los demás compañeros y compañeras, con el objetivo de hacer más competente a cada quien. Al trabajar en equipo, cuando alguien gana, todo el grupo gana. En este caso, todas las personas ganamos en conocimientos y la principal beneficiaria de este conocimiento es la familia, puesto que ella recibirá toda la información necesaria, de su profesional de referencia, para tomar decisiones informadas. Pero, para entender bien ese trabajo transdisciplinar debemos entender que este consiste en ayudar a la familia a reflexionar, a encontrar sus mejores estrategias para conseguir objetivos verdaderamente funcionales para su hijo o hija en su entorno y ayudarles a implementarlas. Entendido esto, debemos dejar a un lado las reticencias a compartir y los miedos al intrusismo. Nunca va a existir intrusismo profesional, pues nunca implementamos técnicas propias de otra profesión. El sujeto profesional especialista en una disciplina sigue siendo experto en su disciplina y utiliza su saber para apoyar a las demás personas, igual que estas le apoyarán cuando lo necesite. Esa transdisciplinariedad y ese apoyo mutuo será utilizado para ofrecer a la familia una respuesta coherente a sus necesidades. Pero esto sólo lo conseguiremos cuando abandonemos nuestro rol de persona experta y entendamos que las únicas personas expertas en la niñez y sus necesidades son su familia. De nada sirven los conocimientos técnicos de cada profesional, si la familia no es capaz de reflexionar sobre las fortalezas de su hija o hijo y las oportunidades de aprendizaje que puede encontrar al cabo del día. Las familias no entienden de áreas o de disciplinas, entienden de sus preocupaciones, de sus necesidades, de sus competencias como cuidadoras principales y su deseo de favorecer el desarrollo de su hijo o hija. Un desarrollo que no pueden fragmentar en áreas, porque el desarrollo es global y la evolución en un área influirá en las otras. Por tanto, como profesionales, necesitamos compartir conocimientos y competencias, saber que tenemos el apoyo de nuestros compañeros y compañeras para ayudarnos a brindar el apoyo que necesitan las familias.

- Los objetivos, ¿los pone cada profesional por separado o todo el grupo a la vez?

Respuesta: No es el sujeto profesional el que marca los objetivos a seguir. Éste acompaña a la familia, dándole información y ayudándole a reflexionar, a fin de que tenga unas expectativas lo más reales y alcanzables posible con respecto al niño o niña. En colaboración estrecha con la familia y a partir de sus prioridades, les ayudamos para que sepan establecer unos objetivos funcionales y adaptados al niño o niña y lograr, así, un aprendizaje significativo. Por tanto, los objetivos los pone la familia en colaboración con cada profesional.

- Si las familias no dejan que vayas a su casa, ¿qué haces?

Respuesta: No es algo que suela ocurrir. Pensar que no van a querer que entremos en su hogar es más un prejuicio profesional que una realidad. Con todo, si las familias no son partícipes de que el sujeto profesional de referencia acceda al domicilio familiar

10 Noelia Orcajada-Sánchez, Elisabet Ortuño-Cano, Conchi Martínez-Pérez y Francisco Alberto García-Sánchez

Los artículos de la Revista Electrónica Educare del Centro de Investigación y Docencia en Educación de la Universidad Nacional, Costa Rica, se comparten bajo términos de la Licencia Creative Commons: Reconocimiento, № Comercial, Sin Obra Derivada 3.0 Costa Rica. Las autorizaciones adicionales a las aquí delimitadas se pueden obtener en el correo: educare@una.cr 
http://doi.org/10.15359/ree.24-3.6

también podemos trabajar. Deberemos tener en cuenta que las PCF se basan en la intervención, apoyo y acompañamiento a la familia en los entornos naturales del niño o de la niña. Del tal modo, si los padres, madres o personas cuidadoras principales del niño o de la niña no son partícipes de que accedamos al domicilio, podríamos buscar la funcionalidad y aprovechamiento de los demás entornos, esperando su competencia y su avance a fin de poder acceder al domicilio de una forma gradual. Estos otros entornos nos podrían servir para valorar o analizar con los padres las estrategias de intervención ante una determinada situación o conducta. No obstante, recordemos que, en la mayoría de los casos, la presencia del niño o niña no será imprescindible, por lo que la sesión con la familia la podremos desarrollar en cualquier lugar que la familia estime oportuno. Esto puede ocurrir, algunas veces, por falta de tiempo, porque la persona cuidadora principal está trabajando y quiere aprovechar el momento del descanso o de la comida para llevar a cabo la sesión. En estos casos, el sujeto profesional puede desplazarse hasta el lugar de trabajo o donde la familia nos indique. Esto es totalmente factible, puesto que se trata de facilitar nuestro acceso a la familia. Con todo ello, es importante insistir en que no nos encontramos con casos en los que las familias nieguen el acceso a su domicilio. Más bien todo lo contrario, saben que cuando profesionalmente se les visita es con el objetivo común de ayudarles en el desarrollo de su hijo o hija y nos abren las puertas de su casa de par en par. Además, muchas veces, es la familia la que nos propone alguna visita en horario que podríamos considerar algo más intempestivo, por ser la hora de la comida, del baño, etc..., pero lo hacen con la intención de que veamos in situ lo que ocurre en esas determinadas situaciones.

En cuanto al Grado en Logopedia las dudas giraron en torno a la implicación de la familia y las herramientas usadas en las PCF:

- En casa, el niño o la niña, ¿no se comportaría de forma diferente con su padre y madre y tú delante?, ¿no sería más difícil que mantenga la atención?

Respuesta: Debemos tener en cuenta que el domicilio es el entorno donde el niño o la niña se relaciona, juega, aprende y experimenta de una forma habitual, es el lugar donde pasa la mayor parte del tiempo junto con el colegio. Por tanto, entenderemos el domicilio como un lugar que le ofrece múltiples oportunidades de aprendizaje, así como mayor motivación y confianza en sí por encontrarse en un lugar que conoce. Y, al igual que ocurre con el domicilio, ocurre en cualquier otro entorno en el que participe, ese es su entorno natural.

También de esta forma deberemos concebir que todo esto hará que su conducta sea diferente a la que normalmente pueda efectuar en la consulta clínica, pero no por ello perjudicará la intervención, sino que de esta forma será más fácil poder ver una conducta 
http://doi.org/10.15359/ree.24-3.6

http://www.una.ac.cr/educare

educare@una.cr

más real y cercana a la vida cotidiana que realiza, lo que nos ayudará a entender, acompañar y apoyar mejor a la familia durante el proceso de toma de decisiones, en el establecimiento de objetivos y en la identificación de estrategias para conseguirlos. No debemos olvidar que la conducta que nos interesa es la del niño o la niña con su familia en su entorno natural, no con nosotros o nosotras como especialistas.

Por supuesto que el niño o la niña, posiblemente, no actúe de la misma manera cuando la familia recibe la visita profesional, pero no porque esté la familia presente, sino porque está el sujeto profesional, y ese es el gran desconocido. Pero no olvidemos que no vamos a trabajar directamente con el niño o la niña, sino con la familia. Vamos a reflexionar con ella y vamos a ayudarle y apoyarle en las decisiones que tome, para que sea la propia familia la que actúe cuando el sujeto profesional no esté presente. Es en ese momento, entre visita y visita profesional, cuando ocurrirá la verdadera intervención, cuando la familia tendrá la oportunidad de poner en marcha las estrategias acordadas para conseguir los objetivos seleccionados. Y será en esos momentos cuando el niño o la niña muestren su propio ser, verdaderamente. Por tanto, poco importa que no se comporte como realmente es cuando hay profesionales delante, lo que nos importa es la información que nos da la familia con el fin de poder analizar y reflexionar conjuntamente sobre las decisiones a tomar.

- Si tienes que enseñar algo a los padres y madres lo haces en el momento, pero... ¿si no está el niño o la niña?

Respuesta: La persona profesional está para apoyar a la familia y ayudarle a encontrar sus propios recursos y estrategias, le ayudamos a reflexionar sobre sus objetivos y cómo conseguirlos. No es imprescindible que el niño o la niña esté presente en la sesión de trabajo con la familia. Pero si en algún momento tenemos que valorar aspectos o situaciones puntuales, o incluso modelar alguna estrategia a seguir, entonces sí deberemos buscar el momento en el que se encuentre el niño o la niña para analizar la situación, conjuntamente con la familia, in situ. Por otro lado, actualmente contamos con herramientas muy útiles para poder ver la situación real y analizarla con la familia. Los móviles son un recurso muy fácil de usar, para que las familias graben cualquier situación en un momento dado y, después, verla, analizarla, reflexionar sobre ella. Además, esas grabaciones ayudan a las familias a ver lo que realmente están haciendo y cómo lo están haciendo. Muchas veces no somos conscientes de lo que hacemos realmente o de cómo estamos actuando ante una determinada situación, hasta que no nos vemos, y es entonces cuando miramos las opciones de mejora. Por tanto, debemos tener claro que la intervención propiamente dicha empieza cuando las familias se

12 Noelia Orcajada-Sánchez, Elisabet Ortuño-Cano, Conchi Martínez-Pérez y Francisco Alberto García-Sánchez

Los artículos de la Revista Electrónica Educare del Centro de Investigación y Docencia en Educación de la Universidad Nacional, Costa Rica, se comparten bajo términos de la Licencia Creative Commons: Reconocimiento, № Comercial, Sin Obra Derivada 3.0 Costa Rica. Las autorizaciones adicionales a las aquí delimitadas se pueden obtener en el correo: educare@una.cr 
quedan solas, sin la visita profesional en el domicilio. Pero tenemos que saber que, cuando la familia necesite que hagamos un modelado, que valoremos una situación, etc., como profesionales podemos coordinarnos con la familia para realizar nuestra visita cuando el niño o la niña esté en casa o, bien, analizarla a través de una grabación.

- ¿Estas prácticas son factibles en cuanto a tiempo y desplazamiento?

Respuesta: Por supuesto. Es cierto que tenemos que desplazarnos y que eso conlleva un tiempo. Pero inicialmente es suficiente con una visita semanal al domicilio, frecuencia que incluso podrá disminuir conforme la familia gane competencias. Pensemos en cuántas sesiones recibe un solo niño o niña al cabo de una semana dentro de un servicio de AT ambulatorio, a manos de diferentes profesionales. Por otro lado, estas prácticas centradas en la familia multiplican las oportunidades de aprendizaje para cada infante. Recordemos el esquema de Jung (2003): por una hora de trabajo con la familia, el niño o la niña podría obtener hasta 84 horas de oportunidades de aprendizaje al cabo de una semana. Sin embargo, cuando actuamos desde unas prácticas ambulatorias, prácticamente solo contamos con la hora de trabajo que el niño o la niña recibe por la parte profesional, ya que desde ese trabajo ambulatorio tenemos mucho menos efectividad a la hora de generalizar oportunidades de aprendizaje en el entorno natural. Es evidente que es factible y que el rendimiento es mucho mayor.

- En el Ecomapa, si aparece una fuente de estrés, ¿harías algo para modificarlo?

Respuesta: Primero tendrá que ser la familia la que plantee que quiere cambiar esa percepción. El sujeto profesional no es fuente de cambio, somos un apoyo para los cambios que la familia quiera conseguir. Por otro lado, hay que aclarar que la aplicación del Ecomapa nos sirve para valorar la red de apoyos de la familia y que la propia familia identifique y sea consciente de esos apoyos. Es una herramienta más de valoración, de obtención de información, no de intervención. Es verdad que en el momento que hacemos reflexionar, ya estamos haciendo cierta intervención, pero debemos tener cuidado con no interpretar y enjuiciar la información que recibimos en un momento de valoración. Y hemos de insistir en que no somos, como profesionales, las personas que tenemos que provocar ningún cambio en cuanto al estrés o al apoyo que cada miembro de la familia percibe sobre algunos de los recursos que surgen en el Ecomapa. Con todo, si alguno de esos apoyos crea tal estrés, a miembros de la familia, que desde la familia se llegue a plantear cambiarlo como una de sus principales preocupaciones, tal vez tengamos que hacer reflexionar con miembros particulares de la familia sobre las posibilidades de cambiar esa situación: ¿realmente está en sus manos conseguirlo?, ¿simplemente debería aceptar la situación? Una vez más, la clave está en la reflexión, en formular a las familias preguntas orientadas, de tal forma que les lleve a reflexionar sobre ello y adoptar la mejor estrategia, la mejor opción. 
- La entrevista basada en rutinas, ¿cómo empieza?, ¿se pregunta todo el día?

Respuesta: La entrevista basada en rutinas es una herramienta concreta, propuesta por determinadas investigaciones, para evaluar las necesidades y prioridades de la familia de cara a planificar la intervención (McWilliam et al., 2009). Obviamente, puede haber otras formas de evaluar esas necesidades y prioridades. Ahora bien, quienes defienden utilizar esta herramienta la plantean como fundamental para implementar un modelo de intervención basado en rutinas (Boavida et al., 2013; McWilliam, 2010, 2016) y con rigurosidad en su protocolo de utilización. Se puede realizar sobre una rutina en concreto, sin necesidad de tener que pasar por todo el día, pero aconsejamos que se haga sólo cuando utilizamos la herramienta en alguna evaluación de seguimiento en donde se detecte el cambio en alguna rutina. Cuando se trata de una valoración inicial, aconsejaríamos realizar una entrevista basada en rutinas que recorra las actividades de todo el día y hacerlo empezando por el primer momento de la mañana, por el despertar, avanzando de forma secuencial. Son muchas las preocupaciones y las oportunidades de aprendizaje que pueden ir surgiendo cuando se describe cada momento del día.

- ¿Cuándo empieza la intervención?

Respuesta: La intervención empieza desde el primer momento cuando la familia se pone en contacto con el recurso de atención temprana. Ya en ese momento estamos escuchando a la familia, cuando nos cuenta por qué nos contacta y nos hace saber sus preocupaciones. Ese acto ya tiene un componente de intervención que puede ser más o menos efectivo e intenso, dependiendo de la forma en que lo hagamos y en la medida en que lo cuidemos. De hecho, quizás sea uno de los momentos más importantes, pues nuestro objetivo, en ese instante, es transmitir a la familia nuestra forma de trabajar. Algo que no es fácil en prácticas centradas en la familia, ya que la AT tiene una amplia trayectoria de corte clínico y ambulatorio. Por tanto, ese es nuestro primer paso en el proceso de intervención, hacer entender a las familias que los principales expertos en su hijo o hija son ellas y ellas serán los que van a tener la capacidad de decidir y proponer objetivos e incluso estrategias para conseguirlo. Dejándoles claro que la intervención profesional de atención temprana de apoyo y colaboración, con disposición a facilitarles toda la información que necesiten para tomar las mejores decisiones y ayudarles a encontrar y ajustar las estrategias para ayudar a su niño o niña en su desarrollo. Ahora bien, a partir de ahí, debemos entender que la puesta en marcha de todas las decisiones tomadas, sobre objetivos a conseguir y estrategias a utilizar, tendrá lugar cuando el sujeto profesional abandone el domicilio y la familia vuelva a su rutina con su pequeño o pequeña. Será entonces cuando la familia desplegará todas las herramientas y los recursos, que el sujeto profesional le ha ayudado a identificar y a saber utilizar, para ser más competente en su labor como padres y madres y para favorecer el desarrollo de su hijo o hija. 
http://doi.org/10.15359/ree.24-3.6

http://www.una.ac.cr/educare educare@una.cr

Analizando las características de las preguntas formuladas encontramos, en los tres grupos, preguntas que denotan una falta de conocimiento sobre la forma de implementar unas PCF en atención temprana:

- Grado en Fisioterapia: falta de conocimiento sobre la forma de valorar las características de la niñez ("...no valoras ni tocas al niño?").

- Grado en Terapia Ocupacional: falta de conocimiento sobre la forma de trabajar en equipo ("¿Dónde quedan las competencias de cada profesional?"); sobre la forma de planificar el trabajo ("Los objetivos, ¿los pone cada profesional por separado...?"); y sobre la operatividad de las visitas a domicilio y viabilidad de su realización ("Si la familia no deja que vayas a su casa...").

- Grado en Logopedia:falta de conocimiento práctico sobreel desarrollo dela intervención en las visitas al domicilio ("¿no se comportaría (el niño, la niña) de forma diferente con su familia y tú delante?"; “...pero, ¿si no está el niño o la niña (presente)...?”; “¿cuándo empieza la intervención?")

En el Grado en Fisioterapia, encontramos también preguntas que denotan la preocupación por la calidad técnica de la intervención. Posiblemente esto se debe a la necesidad, sentida por el alumnado, de intervenir sobre el órgano y su función, lo que les lleva a seguir pensando en la aplicación de técnicas específicas que se entienden "necesarias" (... un padre no va a reproducir una técnica fisioterapéutica como un profesional"; ... si existe un problema estructural ¿lo dejas de tratar?; PCF“....más como un complemento a la intervención clínica que como única opción”).

Las preguntas de este grupo de estudiantes también denotan desconfianza en la capacidad de la familia para ver los problemas reales ("Si la familia no plantea hacer lo mejor para su hijo o hija, o no se implica..."). Así mismo, vemos en sus preguntas un conflicto ético por intervenir lo que como profesional se ve necesario (... lo dejas aunque sepas que no es lo mejor?)

Por su parte, en el Grado en Logopedia también encontramos preguntas centradas en las herramientas utilizadas para la recogida de información en el entorno natural ("... en el ecomapa..."; "la entrevista basada en rutinas...").

\section{Conclusión}

Si nos detenemos un breve instante y analizamos, tanto la temática como el tipo de preguntas realizadas por los grupos de estudiantes de los diferentes grados, lo primero que llama la atención es que diferentes perfiles profesionales plantean dudas también diferentes sobre las PCF. En estas diferencias puede haber influido el planteamiento o filosofía subyacente en la 
http://doi.org/10.15359/ree.24-3.6

http://www.una.ac.cr/educare

educare@una.cr

formación recibida. Esta puede haber sido marcadamente preventiva y clínico/rehabilitadora en el caso del Grado en Fisioterapia, con referencia constante a la profundización en técnicas profesionales específicas. No en vano, en la web de la titulación (Universidad Miguel Hernández, 2018a) se habla específicamente de preparar a los futuros sujetos egresados en Fisioterapia:

Para la promoción y mantenimiento de la salud, y la prevención de las enfermedades y de sus consecuencias, [capacitándolos para] aplicar métodos, procedimientos, técnicas y actuaciones que curan, recuperan y adaptan a personas afectadas de deterioros, limitaciones funcionales, o cambios en la función física y en el estado de salud, producidos por diversas causas. (Párr. 1)

Por su parte, en la web del Grado en Terapia Ocupacional (Universidad Miguel Hernández, 2018b) encontramos referencias menos marcadas a la utilización de técnicas específicas. En concreto, el Grado plantea una formación:

Con el objetivo de maximizar la salud y el bienestar de las personas a lo largo de su ciclo vital, ... [utilizando para ello] la ocupación significativa (actividades de la vida diaria, educación, trabajo, juego, ocio y participación social) con un fin terapéutico. (Párr. 1)

Por último, en la web del Grado en Logopedia (Universidad de Murcia, 2018) solo encontramos una referencia general a que es una titulación "sanitaria ... especializada en la comprensión, el diagnóstico, la intervención y la investigación de los trastornos del lenguaje y la comunicación" (párr. 1).

Además de ello, seguramente también pueda haber influido en las preguntas de los diferentes grupos de estudiantes el grado de profundización al que se ha llegado sobre estas prácticas en cada una de las titulaciones.

Sea como fuere, en el caso del estudiantado de Fisioterapia, su principal preocupación versaba en torno a las dificultades que podría tener la familia a la hora de poner en marcha el proceso de intervención; sus preguntas se caracterizan por tener una base más clínica y se centran en las técnicas a desarrollar y que el alumnado entiende necesarias. Con ello, dicho alumnado está obviando la evidencia científica que señala la menor eficacia de muchas de esas técnicas, en las edades más tempranas, frente a la mayor eficacia de otras formas de intervención que se pueden efectuar en el entorno natural o que, precisamente, requieren la implementación en ese entorno natural, con la implicación activa del niño o la niña y sus personas cuidadoras principales (Novak et al., 2020).

En el caso de estudiantes de Terapia Ocupacional, su interés se centraba especialmente en la transdisciplinariedad y cómo poder desarrollar sus competencias dentro de un equipo de estas características, vieron grandes dificultades a la hora de plantear los objetivos y cómo 
http://doi.org/10.15359/ree.24-3.6

conseguir que la familia identifique esas necesidades y sea capaz de marcarse los objetivos que les den respuesta. Estas preguntas, como otras similares observadas en el alumnado de los Grados de Fisioterapia y Logopedia, denotan desconocimiento sobre la forma de implementar las PCF y esa necesidad profesional de "hacer" más que "dejar hacer" para que la persona cuidadora principal del niño o la niña desarrolle competencias propias y gane confianza en sus acciones (García-Sánchez et al., 2018).

Finalmente, en el caso de estudiantes de Logopedia, se apreciaba un interés especial por profundizar en cómo conseguir la implicación de la familia y las herramientas utilizadas para tal fin. Posiblemente ello fue debido a que era el único grupo de estudiantes que había tenido una asignatura específica de atención temprana en su formación curricular, donde habían podido abordar ya los principios fundamentales de esta práctica de intervención centrada en la familia. Ello apunta hacia la importancia de incluir estos contenidos en la formación curricular de profesionales que tienen la atención temprana como un posible nicho de trabajo.

En cualquier caso, podemos observar que, independientemente del tipo de preguntas o reflexiones planteadas y su grado de profundización, en todos los casos, y como no podía ser de otra manera atendiendo a la temática que nos ocupa, hay un eje común sobre el que gira cualquier cuestión planteada: la familia. Sin embargo, es cierto que en las titulaciones de Fisioterapia y Terapia Ocupacional seguimos percibiendo una orientación especialmente clínica en cualquier aportación de estudiantes. Les preocupa lo que haga la familia, pero les preocupa desde esa perspectiva más técnica que les aporta su propia disciplina. Eso hace que su discurso adopte una posición de persona experta, con el consiguiente temor a perder ese rol.

Sin embargo, en las preguntas planteadas por estudiantes del último curso de Logopedia ya se percibe un mayor acercamiento a esas PCF, puesto que reflexionan sobre las herramientas necesarias para desempeñar su labor como profesionales que apoyan y colaboran con la familia.

Todo ello nos lleva a concluir acerca de la necesidad de reflexionar sobre la formación que están recibiendo actualmente los futuros y futuras profesionales de la atención temprana y la necesaria actualización de esa formación. Estudios previos han evidenciado las necesidades de formación en PCF de profesionales de atención temprana actualmente en ejercicio (García-Sánchez et al., 2018). Deberíamos concretar también cómo llevar a cabo la actualización de la formación de los futuros grupos de profesionales y, para ello, podría ser interesante profundizar, en futuras investigaciones, sobre los conocimientos que posee el estudiantado de último curso de aquellas titulaciones que habilitan para ejercer su profesión dentro del ámbito de la atención temprana, así como las dudas que se plantean al abordar estas temáticas. En este sentido, sería interesante corroborar si las posibles diferencias que existan en las dudas que plantea el estudiantado de las diferentes titulaciones pueden estar mediatizadas por su formación general en la disciplina o solo en función del acercamiento que les hayan ofrecido sus docentes hacia las PCF. 
http://doi.org/10.15359/ree.24-3.6

http://www.una.ac.cr/educare

educare@una.cr

\section{Declaración de Material complementario}

Este artículo tiene disponible como material complementario:

- La versión preprint del artículo en https://doi.org/10.5281/zenodo.3366321

\section{Referencias}

Boavida, T., Aguiar, C. y McWilliam, R. A. (2013). A training program to improve IFSP/IEP goals and objectives through the routines-based interview. Topics in Early Childhood Special Education, 33(4), 200-211. https://doi.org/10.1177/0271121413494416

Dunst, C. J. (2000). Revisiting "Rethinking early intervention." Topics in Early Childhood Special Education, 20(2), 95-104. https://doi.org/10.1177/027112140002000205

Dunst, C. J. (2002). Family-centered practices: Birth through high school. The Journal of Special Education, 36(3), 139-147. https://doi.org/10.1177/00224669020360030401

Dunst, C. J. (2017). Procedures for developing evidence-informed performance checklists for improving early childhood intervention practices. Journal of Education and Learning, 6(3), 1-13. https://doi.org/10.5539/jel.v6n3p1

Dunst, C. J., Trivette, C. M. y Hamby, D. W. (2007). Meta-analysis of family-centered. helpgiving practices research. Mental Retardation and Developmental Disabilities Research Review, 13(4), 370-378. https://doi.org/10.1002/mrdd.20176

Federación Estatal de Asociaciones de Profesionales de Atención Temprana. (2000). Libro blanco de la atención temprana. Real Patronato sobre Discapacidad Intelectual.

Forry, N. D., Moodie, S., Simkin, S. y Rothenberg, L. (2011). Family-provider relationships: A multidisciplinary review of high quality practices and associations with family, child, and provider outcomes (Issue briefOPRE 2011-26a). Office of Planning, Research and Evaluation, Administration for Children and Families, U.S. Department of Health and Human Services.

García-Sánchez, F. A., Escorcia Mora, C. T., Sánchez-López. M. C., Orcajada Sánchez, N. y Hernández-Pérez. E. (2014). Atención temprana centrada en la familia. Revista Siglo Cero, 45(3), 6-27. https://sid.usal.es/articulos/discapacidad/20896/8-2-6/atencion-tempranacentrada-en-la-familia.aspx

García-Sánchez, F. A., Rubio-Gómez, N., Orcajada-Sánchez, N., Escorcia-Mora, C. T. y CañadasPérez, M. (2018). Necesidades de formación en prácticas centradas en la familia en profesionales de atención temprana españoles. Bordón. Revista de Pedagogía, 70(2), 39-55. 
http://doi.org/10.15359/ree.24-3.6

http://doi.org/10.13042/Bordon.2018.59913

Jung, L. A. (2003) More better: Maximizing natural learning opportunities. Young Exceptional Children, 6(3), 21-26. https://doi.org/10.1177/109625060300600303

McWilliam, R. A. (2010). Routines-based early intervention. Brookes Publishing.

McWilliam, R. A. (2016). The Routines-Based Model for supporting speech and language. Revista de Logopedia, Foniatría y Audiología, 36(4), 178-184. doi.org/10.1016/j.rlfa.2016.07.005

McWilliam, R. A., Casey, A. M. y Sims, J. (2009). The routines-based interview. A method for gathering information and assessing needs. Infants and Young Children, 22(3), 224-233.

Novak, I., Morgan, C., Fahey, M., Finch-Edmondson, M., Galea, C., Hines, A., Langdon, K., Mc Namara, M., Paton, M. C. B., Popat, H., Shore, B., Khamis, A., Stanton, E., Finemore, O. P., Tricks, A., Velde, A. T., Dark, L., Morton, N. y Badawi, N. (2020). State of the evidence traffic lights 2019: Systematic review of interventions for preventing and treating children with cerebral palsy. Current Neurology and Neuroscience Reports, 20(2), 1-21. http://doi. org/10.1007/s11910-020-1022-z

Orcajada Sánchez, N. (2017). Diseño, aplicación y evaluación del plan de formación de tutores de atención temprana [Tesis doctoral]. Universidad de Murcia, Murcia. https://digitum.um.es/ xmlui/bitstream/10201/53422/1/TESIS Noelia\%200rcajada.pdf

Ruiz-Veerman, E., Gutiez Cuevas, P. y Arizcun-Pineda, J. (2005). La formación de los profesionales de atención temprana. En P. Gutiez Cuevas (Coord.), Atención temprana. Prevención, detección e intervención en el desarrollo (0-6 años) y sus alteraciones (pp. 935-953). Editorial Complutense.

Universidad de Murcia. (2018). Facultad de Psicología. Grado en Logopedia. https://www.um.es/ web/psicologia/contenido/estudios/grados/logopedia

Universidad Miguel Hernández. (2018a). Grado de Fisioterapia. Plan 2018. www.umh.es/ contenido/PDI/:tit g 264 M1/datos es.html

Universidad Miguel Hernández. (2018b). Grado de Terapia Ocupacional. Plan 2018. https://www. umh.es/contenido/pdi/:tit g $265 \mathrm{M} 1 /$ datos es.html 